\title{
Automated Sampling In Aceordance with USP
}

Christian G. Müller

ERWEKA GmbH

Heusenstamm, Germany

\section{Introduction}

Fast and complete release of the active substance is the prerequisite for bioavailability of immediate-release oral drug products, whereas a constant and unaffected dissolution is required for controlled-release formulations. Drug release is usually tested in vitro. If in vitro methods are "verified" by in vivo data, they can even be used as surrogates for clinical trials [1]. Investigations on dissolution are important in any stage of a drug product's life cycle. From the early phase of technological development to scaling-up procedures and quality control of marketed batches, meaning ful in vitro data are necessary for the evaluation of possible differences in drug release in regard to drug product safety and efficacy. Moreover, results of dissolution tests are required to prove constant biopharmaceutical quality of marketed products, e.g., after postapproval changes [2].

Dissolution methodology is conventional and is described very precisely in the pharmacopoeias (e.g. Ph. Eur II, PJ 12, and USP 23). In general, precisely working analytical instruments and methods are a prerequisite for the discrimination of differences between pharmaceuticals. In particular; the dissolution equipment must be in accordance to the specifications of pharmacopoeias. In addition, unwanted effects of dissolution devices (e.g. vilbrations) bave to be avoided. Therefore, analytical validation must include the proof of reproducibility for dissolution conditions. The most common way to perform this part of the validation is by checking mechanical parts of the apparatus by gauging and by performing the apparatus suitability test as it is described in USP 23 [3]. Standards in the metal processing industry in the US and the European Union are not completely identical. Therefore, minor differences between European and American dissolution equipment still exist. By careful validation it bas to be proven that those variations bave no effect on the results of dissolution testing [4].

Further influences on the results of a dissolution test can be caused by the performance of the test itself. For instance, it is well known that the size [5] and the positioning [6] of permanently inserted thermometers and sampling probes in the vessels can bave an impact on the bydrodynamics in the vessel and bence on the dissolution bebavior of the dosage form under investigation. When samples are withdrawn manually and probes are only inserted immediately prior to the sampling time point, interferences are avoided. Interferences are to be expected with automated sampling where probes usually stay permanently in the vessels throughout the whole test.

\section{Pharmacopeial Requirements}

ccording to the USP 23 proce-
dure for dissolution testing of
depicted in figure 1. 1 capsules, uncoated tablets, and plain coated tablets samples of dissolution media have to be withdrawn from a zone midway between the surface of the dissolution medium and the top of the rotating basket or blade, not less than $1 \mathrm{~cm}$ from the vessel wall. This positioning of the sampling probes is required for the use of apparatus 1, the basket-apparatus and apparatus 2, the paddle-apparatus. In addition, apparatus 5 , paddle over disk and apparatus 6, Figure 1. Different sampling zones at variable dissolurotating cylinder, require identical positioning of sampling devices as described for apparatuses 1 and 2 . At the same time, a distance of 25 $\pm 2 \mathrm{~mm}$ between the stirring device (basket, blade, cylinder) and the inside bottom of the vessel or disk assembly has to be maintained. By this way the lower limit for adjusting the position of the probes is generally defined, whereas the upper limit varies according to the volume of dissolution medium in the vessel. This implies an adjustment of the probes' position "exactly midway" depending on the

\section{Automated Sampling in Accordance with USP $\mathbf{2 3}$ Requirements}

The ERWEKA DT 80 dissolution-tester is designed to meet the pharmacopeial requirements for sampling as described above. Automatically, at the beginning of each run the $25 \mathrm{~mm}$-difference between the stirring 


\section{Automated Sampling. .. .cont.}

devices and the hemispherical bottom of the vessel is adjusted. This procedure is performed for each individual spindle in order to compensate for small differences in the vessels' geometry. Sampling probes and thermometers are mounted to a holder, which is moved according to the analyst's programmed specifications. The analyst programs the volume of dissolution media and the sampling time intervals into the system's microcomputer. The sampling position is then adjusted by the machine. During each test run the real sampling times are documented together with the temperature in each vessel and the actual revolution speed at the time of sampling. By this way the requirements of USP 23 are exactly met for each run and documented according to good analytical practice.

Other systems have been described earlier with system defined sampling positions. With system defined sampling positions, adjustment of the sampling position, as required by USP, cannot be performed if the volume of dissolution media is varied. Since a variation of the sampling position is not possible, concordance with compendial sampling has to be validated for each volume and drug product under investigation. According to the draft of the FIP Dissolution Guideline [7], the way to prove that any variation of compendial dissolution testing has no effect is by cross-validation. This cross-validation cannot be performed in a general way but needs to be performed for each individual product as described by Schäuble [8].

\section{Conclusion: Redurtion of Work Load}

Withdrawal of dissolution samples is exactly described in the USP 23. Elegant devices for sampling are now available [8]. When the automated sampling devices are not completely in accordance with the pharmacopeial requirements, cross-validation is necessary for each individual product and/or method. Some new programmable automated sampling systems like the ERWEKA DT 80 dissolution tester are completely in accordance with USP 23 which eliminate the need for crossvalidation and reduce the overall workload in the validation of dissolution testing.

Correspondence Address:

Christian G. Müller

ERWEKA GmbH

Ottostrasse 20-22

63150 Heusenstamm

Germany

\section{Literature}

[1]The United States Pharmacopeia, 23rd Edition, Chapter <1088>, USPC, Rockville, 1995

[2] FDA, Center for Drug Evaluation and Research: Guidance for Industry, Immediate Release Solid Oral Dosage Forms. Scale-up and Postapproval Changes, November 1995

[3] The United States Pharmacopeia, 23rd Edition, Chapter <711>, USPC, Rockville, 1995

[4] Krämer, J.: European Perspective-American and European Equipment Calibration Issues. AAPS Workshop: Dissolution, Calibration, and Testing, Arlington, September 28/29, 1995

[5] Wells C.E.: Effect of Sampling Probe Size on the Dissolution of Tableted Drug Samples. J. Pharm. Sci., 70, 2 (1981), 232-233.

[6] Savage T.S., Wells C.E.: Automated Sampling of In Vitro Dissolution Medium: Effect of Sampling Probes on Dissolution Rate of Prednisone Tablets. J. Pharm. Sci., 71, 6 (1982), 670-673. [7] Siewert M.: New FIP Guideline for Dissolution Testing of Solid Oral Products. Dissolution Technologies, August 1996, 3-7

[8] Schäuble T.: A Comparison of Various Sampling Methods for Tablet Release Tests Using the Stirrer Methods [USP Apparatus 1\&2]. Dissolution Technologies, May 1996, 11-15. 